\title{
$\mathfrak{J} \mathfrak{n} \mathfrak{h} \mathfrak{a} \mathfrak{l} \mathfrak{t}$
}

Sette

Einleitung

1. Sou $\mathfrak{p} t \mathfrak{f t} \ddot{u} \mathfrak{d}$.

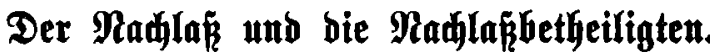

I. Der Nahla eines Berjtorbenen als (Gegenjtand ber Grbfolge. $§ 1$. 5

II. Die Erbfolge.

1. Nothronbigfeit und unmittelbarteit ber Erbfolge. $\$ 2, \ldots, 10$

2. Borausfegungen ber Crbjolge. $\$ 3$. . . . . . . . . . 12

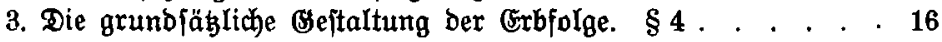

4. (Exbrecht im fubjeftiven Sinne. $\$ 5$. . . . . . . . . . . . . 18

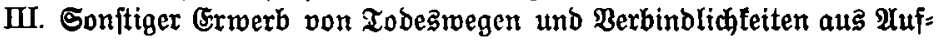

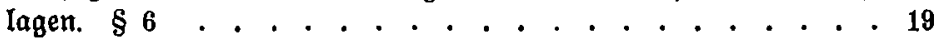

2. $\mathfrak{E} \mathfrak{a} \mathfrak{u} \mathfrak{p}$ t $\mathfrak{t}$ ü $\mathfrak{x}$.

Die am Madhla er erbredttlid Betheiligten im Cinzelnen.

1. $\mathfrak{A b}\{\boldsymbol{d} \mathfrak{n}$ itt.

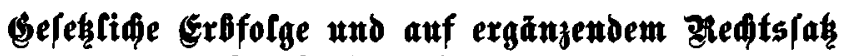
Bernflender Eimzelerwerb.

1. Siapifer. Gereg̨ftife Exbfofge.

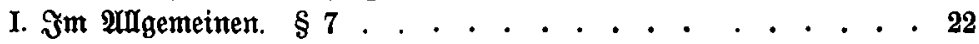

II. Die Crbfolge ber Bermanbten.

1. Berechtigung zur Bermandtenerbfolge. $\$ 8$. . . . . . . 25

2. Die Erbfolge ber Bermanbten im Einzelnen. $\$ 9 . \ldots . . .27$

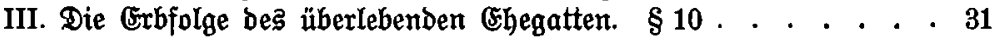

IV. Dex Fistus als gejeblicher (Erbe. $\$ 11$. . . . . . . . . . 33

V. (Exhbhung bes gejeblichen Exbtheils burch Megfall von Mit= erben. $\$ 12$. . . . . . . . . . . . . . . . . . . . . 34

VI. Die $\mathfrak{A}$ แล̆

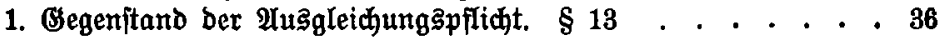

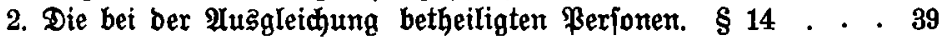

3. Die Durffführung Der $\mathfrak{A} u g h$ leichung. $\$ 15$. . . . . . . . 42

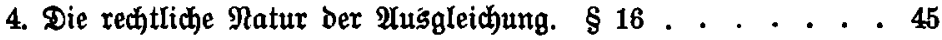

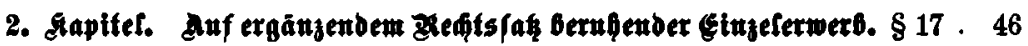




\section{2. $\mathfrak{A} \mathfrak{b} \not \mathbf{w i t t}$.}

Seite

\section{\$̧erfĩ guttgen vou Zobeswegen.}

1. \&apitef. Angemetues. $\$ 18 \ldots \ldots$

2. Eapifer. Das Geftament.

I. Exridhtung Des Tejtaments.

A. Fähigfeit zur crrichtung. $\$ 19$. . . . . . . . . . . 50

B. Form.

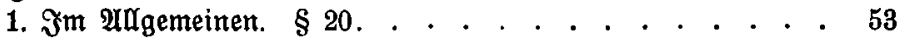

2. Die orbentlichen Reftamentaformen. $\$ 21 . . . \quad . \quad . \quad 55$

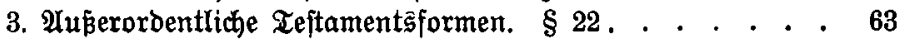

II. Inhalt bes rejtaments.

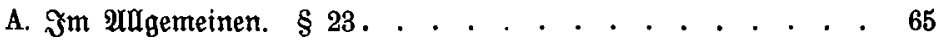

Fortjekung (bie Bumenbungen). $\$ 24$. . . . . . . . . 71

Fortjebung (Bedingungen und Befrijtungen). $\$ 25 . \ldots . \quad . \quad 76$

B. (Erbeinfegung. $\S 26$. . . . . . . . . . . . . . . . 86

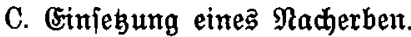

1. Borauşęungen ber Racherbfolge. $\$ 27$. . . . . . . 95

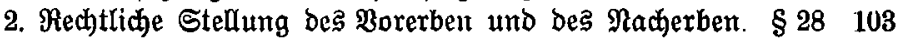

D. Bermächtniffe.

1. Эm $\mathfrak{a}$ gemeinen.

a) Die ßexpnen bes \$ermäctnifjes. § 29 . . . . . . . 115

b) Schranfen bex Wirffamfeit Der Bermächtniffe. $\$ 30 . \quad 122$

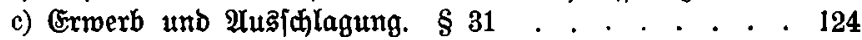

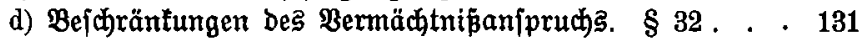

2. Bermähtniß eines bejtimmten Begenjtandes.

a) Dex Begenftand gehb̈rt bem Erblafier. $\$ 33$. . . . 133

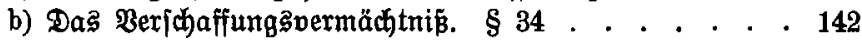

3. Bermäđhtní̄ einer nur, ber Sattung nach beftimmten

Sache. §35. . . . . . . . . . . . . . . . . . 145

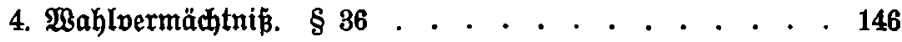

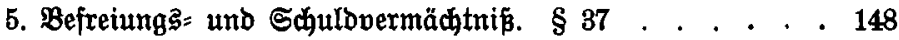

E. 2luflagen. $\S 38$. . . . . . . . . . . . . . . . . 149

F. Tejtamentsoollitrecter.

1. Eiftorifbes. $\$ 39$. . . . . . . . . . . . . . . 152

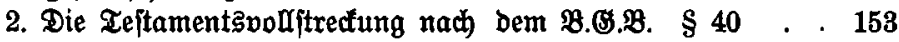

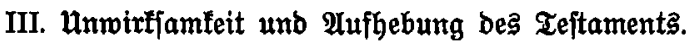

A. Unwirffamfeit. $\S 41$. . . . . . . . . . . . . . . 164

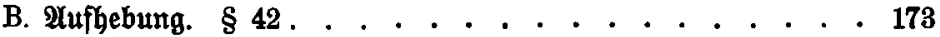

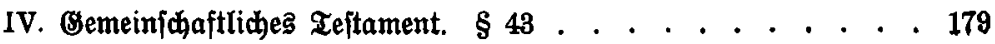




\section{Stapifef. Det Grbpertrag.}

Sette

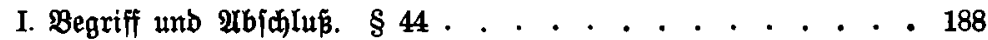

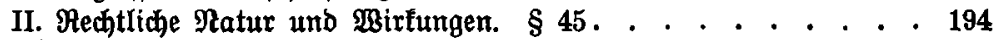

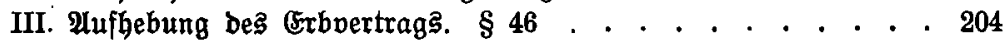

IV. Der gegenfeitige Erbvertrag. $\$ 47$. . . . . . . . . . 211

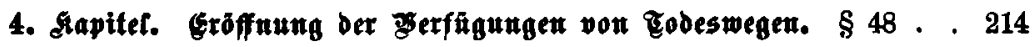

3. $\mathfrak{A} \mathfrak{b}\{\dot{m} \mathfrak{n i t t}$.

\section{Zflidittgeirstedf.}

I. Die grumbjäglide (Sejtaltung bes ßfichttheilsrechtes.

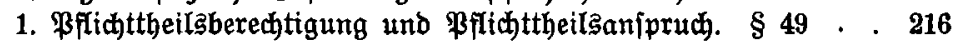

2. Das Bflidttheilšxecht ber entfernteren Abtömmlinge und ber EItern. $\$ 50$. . . . . . . . . . . . . . . . . . 220

3. Der gejegliche (5xbtheil als Brunblage ber \$flichttheiraberech= nung. $\$ 51$. . . . . . . . . . . . . . . . . . . 230

4. Dex Betrag des Pflichttheils. \&52 . . . . . . . . . . 233

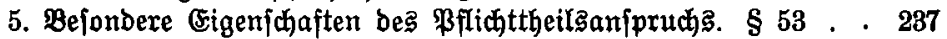

6. Die Pflichthcilalaft. $\S 54$. . . . . . . . . . . . 241

II. Die Berüđfichtigung von ßorempfängen.

1. Unrechnung auf ben Pflichttheil. $\$ 55$. . . . . . . . . 244

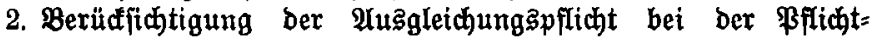

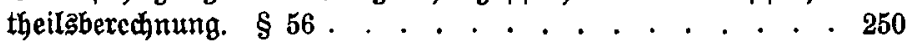

III. Cntżehung und Bejđränfung des \$flichttheils. § 57 . . . 265

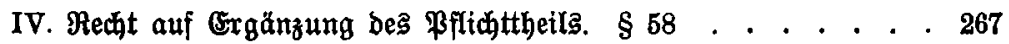

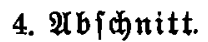

Graberzint.

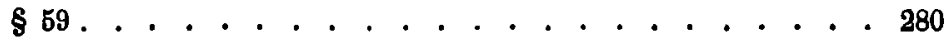

5. $\mathfrak{A} \mathfrak{b}\lceil\not{d} \mathfrak{i t t}$.

Exbutwãroigkeit.

$\S 60 . . \ldots . . . . . . . . .291$

3. $\mathfrak{g} \mathfrak{a} \mathfrak{u p t} \mathfrak{f t} \mathfrak{u} \mathfrak{d}$.

Crwerb ber Grbifant.

1. $\mathfrak{A} \mathfrak{b}\{\mathfrak{d} \mathfrak{n}$ itt.

Aunafime und Ausfaffagung.

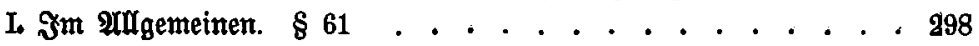

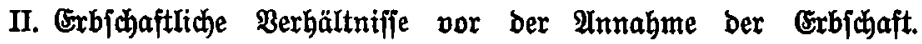
$\S 62 \ldots . . \ldots . . . . . . .811$ 
2. $\mathfrak{A} \mathfrak{b}\lceil\mathfrak{d} \mathfrak{n} i t t$.

Sette

\section{Die redflide Gterlung bes Erben.}

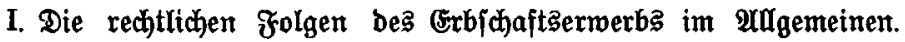

$\S 63$.

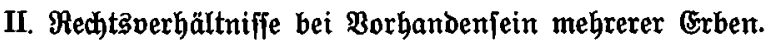

1. Die Erbengemeinfdaft. $\$ 64$. . . . . . . . . . . . 338

2. Alfhebung Der (Erbengemeinichaft. $\S 65$. . . . . . . . . 349

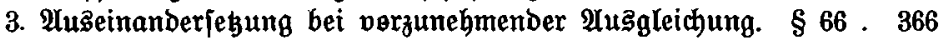

III. (Fibid)ein und rejtamentsvollitrederzeugnis.

1. (Extheilung, (Eingiehung und Araftlosertlärung bes (5rbłheins.

$\$ 67$. . . . . . . . . . . . . . . . . . . . . . 370

2. Rechtliche Bedeutung bes (Erbicheing. $\$ 68$. . . . . . . 375

3. Teptamentspoujtredferzeugniß̧. $\$ 69$. . . . . . . . . . 392

\section{4. Şa uptftü $\mathfrak{x}$.}

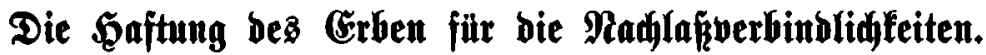

\section{1. $\mathfrak{A b}\{\mathfrak{c} \mathfrak{n}$ itt.}

\section{Einfeitendes.}

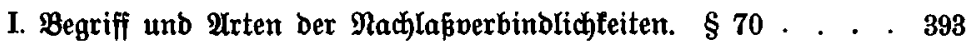

II. DaS ßroblem Der Grbenhaftung. $\$ 71$. . . . . . . . . . 403

\section{2. $\mathfrak{A b}\lceil\dot{c} \mathfrak{n}$ itt.}

\section{Goftung bes Alteiterben.}

I. Die \$aftungsgrundfäge. $\$ 72$. . . . . . . . . . . 405

II. Die Эnventarerrichtung in ihrer Bebeutung für bie ఏaftung Des

Erben. $\$ 73 \ldots . . . . . . . . .4414$

III. Die Saftung beß Crben im Cingelnen.

1. Auffdiebende (sinreben. $\$ 74$. . . . . . . . . . 433

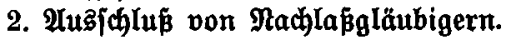

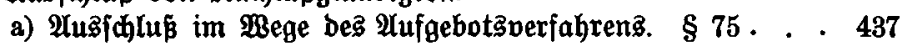

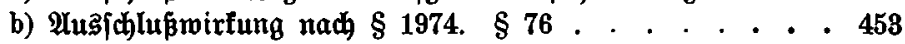

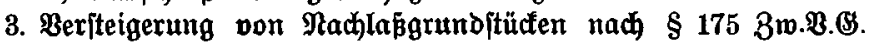

$\S 77$. . . . . . . . . . . . . . . . . . . . . . 457

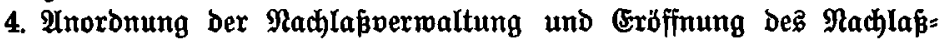
fonfuries.

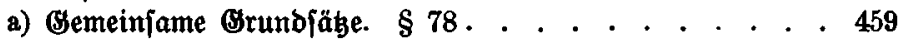

b) ఇachlaßsverwaltung. $\$ 79$. . . . . . . . . . . . 468

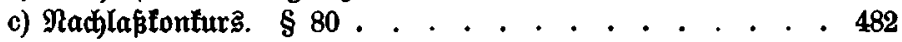

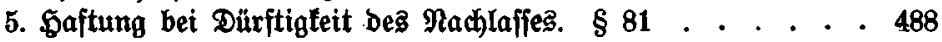

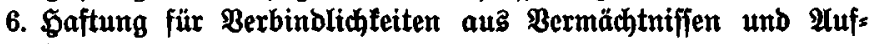

lagen. $\$ 82 \ldots . . . . . . . . . . . .497$ 
Sette

7. Saftung in befonberen ₹ällen.

a) Saftung bes Nacherben und Des \$orexben nach Dem Falle ber গlacherbfolge. $\$ 83$. . . . . . . . . . . . . . . . . 498

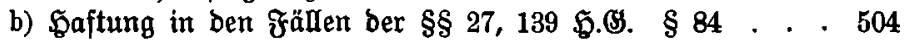

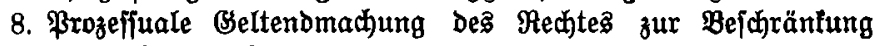
ber \$aftung. $\$ 85$. . . . . . . . . . . . . . . . . 506

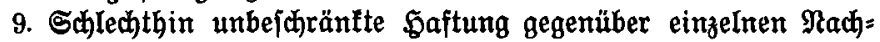

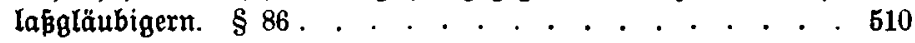

3. $\mathfrak{A b}\{\boldsymbol{c} \mathfrak{n} i t t$.

\section{foaftung mefreter srben.}

I. Die Şauptgrundjäge.

1. Cinleitenbes. $\$ 87$

2. Saftung vor ber Igeilung. $\$ 88$. . . . . . . . . . . 515

3. Faftung nah ber Theilung. $\$ 89$. . . . . . . . . . 520

II. Befondere Fălle.

1. Waftung bei Berufung eines (5xben zu mehreren Grbtheilen. $\$ 90$. . . . . . . . . . . . . . . . . . . . . , 530

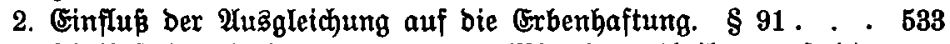

3. Einflus ber Uebertragung von Miterbenantheilen auf bie Erbenhaftung. $\S 92 . . . . . . . . . . . . . .536$

5. Să

\section{Sthuth bes Grben.}

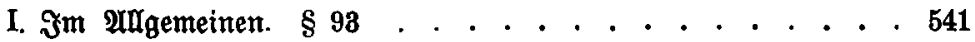

II. Crbiðafţanipruch).

1. Die \$arteien. $\$ 94$. . . . . . . . . . . . . . . . 545

2. Serausgabepflictt. $\$ 95$. . . . . . . . . . . . . 554

II. Cinzelanfprüche. $\S 96$. . . . . . . . . . . . . . 571

6. Şa $\mathfrak{u} \mathfrak{p} t \mathfrak{t} \mathfrak{t} \mathfrak{u} \mathfrak{t}$.

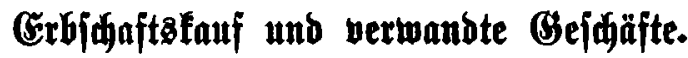

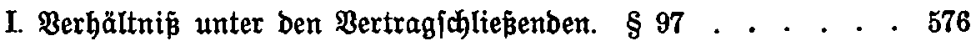

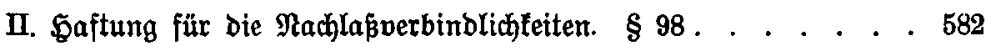
Sadifegifter. . . . . . . . . . . . . . . . . 585 
